\title{
Biological characterization of three immortalized esophageal epithelial cell lines
}

\author{
FA-MIN ZENG ${ }^{1,2}$, YANG-MIN XIE ${ }^{1,3}$, LIAN-DI LIAO $^{1,4}$, LI-YAN LI $^{1,4}$, \\ BO $\mathrm{CHEN}^{1,4}$, JIAN-JUN XIE ${ }^{1,2}$, LI-YAN XU ${ }^{1,4}$ and EN-MIN LI ${ }^{1,2}$ \\ ${ }^{1}$ Key Laboratory of Molecular Biology for High Cancer Incidence in The Coastal Chaoshan Area; \\ ${ }^{2}$ Department of Biochemistry and Molecular Biology; ${ }^{3}$ Experimental Animal Center and ${ }^{4}$ Institute of Oncologic Pathology, \\ Medical College of Shantou University, Shantou, Guangdong 515041, P.R. China
}

Received October 12, 2015; Accepted September 2, 2016

DOI: $10.3892 / \mathrm{mmr} .2016 .5813$

\begin{abstract}
The key molecular events that contribute to tumorigenesis are incompletely understood. The aim of the present study was to characterize and compare the biological phenotypes of three human telomerase reverse transcriptase (hTERT) and/or human papillomavirus 16 E6 and E7-immortalized esophageal epithelial cell lines, NE2-hTERT (NE2), NE3-E6E7-hTERT (NE3) and NEcA6-E6E7-hTERT (NEcA6). The present study used soft-agar colony formation assays, tumorigenicity assays in nude mice, and cell proliferation, adhesion and migration assays to identify the biological characteristics of NE2, NE3 and NEcA6 cells. NE2 and NE3 cells exhibited characteristics of benign cells, such as the inability to grow in soft agar or form tumors in nude mice. By contrast, NEcA6 cells had undergone transformation, as demonstrated by the ability to grow in soft agar and form tumors in nude mice. In addition, NEcA6 cells exhibited increased migration and adhesion capabilities when compared with NE2 and NE3 cells. In order to identify mechanism(s) that may contribute to the altered biological phenotypes exhibited by these cells, the expression of three proteins involved in modulating cell migration [fascin, ezrin/radixin/moesin family proteins and phosphorylated-focal adhesion kinase (Tyr 397)], as well as the expression status and subcellular localization of three key focal adhesions components (paxillin, talin and kindlin-2) were examined. Paxillin, talin
\end{abstract}

Correspondence to: Professor En-Min Li, Department of Biochemistry and Molecular Biology, Medical College of Shantou University, 22 Xinling Road, Shantou, Guangdong 515041, P.R. China

E-mail:nmli@stu.edu.cn

Professor Li-Yan Xu, Institute of Oncologic Pathology, Medical College of Shantou University, 22 Xinling Road, Shantou, Guangdong 515041, P.R. China

E-mail:1yxu@stu.edu.cn

Key words: immortalized esophageal epithelial cell, tumorigenic potential, cell migration, cell adhesion, cell proliferation and kindlin-2 were localized to adhesive sites that connect F-actin with the extracellular matrix in transformed NEcA6 cells, but were distributed in a diffuse manner in NE2 and NE3 cells. Knockdown of kindlin-2 in NE3 and NEcA6 cells decreased cell adhesion, however, NEcA6 cells demonstrated a greater sensitivity to knockdown of kindlin-2. No significant differences were observed in the protein expression levels of fascin, exrin/radixin/moesin and p-FAK in the three cell lines. In conclusion, these results demonstrate that these three focal adhesion components, particularly kindlin-2, may contribute to the carcinogenesis of esophageal squamous cells.

\section{Introduction}

Cell migration is a crucial cellular function that contributes to a number of important physiological and pathological processes, such as vascular disease and chronic inflammatory diseases including cancer (1-3). Therefore, understanding the fundamental mechanisms underlying cell migration may provide novel and effective therapeutic approaches for the treatment of these diseases, particularly malignant tumors (4). Cell adhesion and migration are important factors involved in the transition of tumors from a non-malignant to a metastatic phenotype (5). An important cellular response to a migration-promoting agent is the extension of protrusions in the direction of migration, with the assembly of focal adhesions at the leading edge and their disassembly at the trailing edge (1). Focal adhesions are large protein complexes located at the basal surface of cells that mediate the physical connection between the extracellular matrix (ECM) and the actin-based cytoskeleton within the cell (6). An important cellular response to a migration-promoting agent is the extension of protrusions in the direction of migration, with the assembly of focal adhesions at the leading edge and their disassembly at the trailing edge (7). Focal adhesions are large protein complexes located at the basal surface of cells that mediate the physical connection between the extracellular matrix (ECM) and the actin-based cytoskeleton within the cell (6). They are speculated to serve critical roles in numerous cell functions, particularly migration and adhesion (8). Focal adhesions consist of heterodimeric transmembrane matrix receptors, known as integrins, which interact with a complex consisting of intracellular structural 
proteins and the actin cytoskeleton through their cytoplasmic tails. In addition, multiple regulatory proteins, including paxillin, talin and kindlin, are located within focal adhesion sites and serve to transduce extracellular signals inside cells, leading to modulation of adhesion and migration (9). First described 20 years ago, paxillin was initially characterized as a 68-kDa focal adhesion protein that undergoes a significant increase in tyrosine phosphorylation upon $\mathrm{v}$-src expression, and is one of the prototypical adaptor proteins involved in integrin signaling $(10,11)$. Paxillin is a scaffold for the recruitment of multiple signaling proteins to the plasma membrane, such as the focal adhesion kinase (FAK) and Src tyrosine kinases. These proteins are activated through the engagement of integrin molecules by ECM ligands, such as fibronectin and collagen $(12,13)$. On the internal plasma membrane, integrins, cytoskeletal-associated proteins and FAK are connected to the actin cytoskeleton via talin and kindlin, which are two structurally and functionally associated protein families that are essential for integrin activation and integrin-mediated signaling (14).

The immortal esophageal epithelial cell lines NE2-hTERT (NE2), NE3 E6E7-hTERT (NE3) and NEcA6-E6E7-hTERT (NEcA6), which were immortalized by overexpression of the human telomerase reverse transcriptase (hTERT) and/or human papillomavirus (HPV) 16 E6 and E7 proteins, are valuable models to study the process of cancer cell immortalization (15-17); an essential pre-requisite to malignant transformation and an initial step in carcinogenesis. These immortalized cell lines have been demonstrated to exhibit a high proliferation rate, but failed to induce colony formation in soft agar (18-20). However, there is limited information regarding the cell migration and adhesion capabilities of these cells. Through a detailed characterization of NE2, NE3 and NEcA6 cell lines, the aim of the present study was to investigate the cell migration and adhesion properties of these cell lines and to identify the molecular mechanisms that contribute to esophageal carcinogenesis.

\section{Materials and methods}

Cell lines and culture conditions. The immortalized human esophageal epithelial cell lines NE2, NE3, and NEcA6 were donated by Professor Sai-Wah Tsao (Department of Anatomy, University of Hong Kong, Hong Kong, China). These cell lines were cultured in a 1:1 mixture of defined keratinocyte serum-free medium (dKSFM; Gibco; Thermo Fisher Scientific, Inc., Waltham, MA, USA) and EpiLife medium (Cascade Biologics, Inc., Portland, OR, USA) in 5\% $\mathrm{CO}_{2}$ at $37^{\circ} \mathrm{C}$.

Characterization of short tandem repeats (STRS). In order to characterize STRs in immortalized esophageal epithelial cell lines, genomic DNA was extracted using the DNA IQ System kit (Promega Corporation, Madison, WI, USA) according to the manufacturer's instructions, and subject to amplification by polymerase chain reaction in a final reaction volume of $25 \mu \mathrm{l}$ using the PowerPlex 18D System (Promega Corporation). Collection and analysis of the data were performed using Data Collection software and GeneMapper software (version 3.2; Applied Biosystems; Thermo Fisher Scientific,
Inc.), respectively. The experiments were performed by Land Huagene Biosciences Co., Ltd. (Guangzhou, China).

Oligonucleotide transfection. Kindlin-2 small interfering RNA (siRNA) and the corresponding non-targeting control RNAs were purchased from Qiagen, Inc. (Valencia, CA, USA). The kindlin-2 siRNA sequence was 5'-CTGGTGGAG AAACTCGATGTA-3'. Oligonucleotide transfections were performed using Lipofectamine ${ }^{\circledR}$ RNAiMAX Transfection Reagent (Thermo Fisher Scientific, Inc.) according to the manufacturer's instructions.

Soft-agar colony formation assay. Cells were seeded on 24-well plates at a density of $2 \times 10^{4}$ cells/well in a 1:1 mixture of dKSFM and EpiLife and $0.7 \%$ agar, with a lower solidified base layer consisting of $0.35 \%$ agar. Colony formation was visualized and quantified using a Leica DMI3000B inverted phase contrast microscope (Leica Microsystems $\mathrm{GmbH}$, Wetzlar, Germany) at 3 weeks after the cells were seeded. Colonies composed of $>16$ cells were counted at x 200 magnification, according to the procedures described previously (20). The mean value was calculated from data obtained from three independent experiments.

Tumorigenicity assays in nude mice. All animal experiments were conducted with the approval of the Institutional Animal Care and Use Committee of Shantou University (Shantou, China). A total of 14 male $\mathrm{Nu} / \mathrm{Nu}$ mice (age, 4-5 weeks) were purchased from Beijing Weitong Lihua Experimental Animal Technical Co., Ltd. (Beijing, China). Animals were maintained at a constant temperature $\left(26^{\circ} \mathrm{C}\right)$ under a light/dark cycle $(14 / 10 \mathrm{~h})$, and had ad libitum access to food and water. Cells (1x10 6 cells/inoculation) were diluted in $100 \mu \mathrm{l}$ PBS and injected subcutaneously into the left and right side of the hip areas of nine $\mathrm{Nu} / \mathrm{Nu}$ mice. Three groups of three mice were inoculated with NE2, NE3 or NEcA6 cells. Animals were palpated twice a week for the appearance of tumors. Mice were sacrificed by ether inhalation at 6 weeks following inoculation, and all tumors were excised, fixed in formalin, embedded in paraffin and cut into sections. Tumors formed by NEcA6 cells were subject to hematoxylin-eosin and immunohistochemical staining. Immunohistochemical staining was performed as described previously (21), using the mouse anti-pan-cytokeratin AE1/AE3 antibody (1:200; cat. no. ZM-0069; ZSGB-Bio, Beijing, China). The tumorigenicity of NEcA6 cells was confirmed by injection of $1 \times 10^{6}$ cells into the front, and the left and right sides of the hip areas of five additional male $\mathrm{Nu} / \mathrm{Nu}$ mice. Each mouse had four injection points.

Cell proliferation assay. Cell proliferation was assessed using the xCELLigence Real-Time Cell Analysis (RTCA) DP instrument (Roche Applied Science, Mannheim, Germany), which was placed in a humidified incubator at $37^{\circ} \mathrm{C}$ and $5 \% \mathrm{CO}_{2}$. The cell proliferation assay was performed as described previously (22). Briefly, $1 \times 10^{4}$ cells were seeded in 16-well microtiter E-plates (Roche Applied Science). Cell impedance was monitored every 15 min for $\sim 48 \mathrm{~h}$ by measuring the cells adhered to the gold metallic plates. After plotting the impedence (cell index) against time (h), the rate of cell impedance (slope) was calculated using the following calculation: Slope $=$ impedance 
Table I. Primary antibodies used in the present study.

\begin{tabular}{|c|c|c|c|c|}
\hline Name & Dilution & $\begin{array}{l}\text { Catalog } \\
\text { number }\end{array}$ & $\begin{array}{l}\text { Clone } \\
\text { number }\end{array}$ & Source \\
\hline Mouse anti-FAK & $1: 500$ & 610088 & 77 & BD Biosciences, Franklin Lakes, NJ, USA \\
\hline Mouse anti-paxillin & $1: 50$ & 610620 & 165 & BD Biosciences \\
\hline Mouse anti-talin & $1: 500$ & T3287 & $8 \mathrm{~d} 4$ & $\begin{array}{l}\text { Sigma-Aldrich; Merck Millipore, } \\
\text { Darmstadt, Germany }\end{array}$ \\
\hline Rabbit anti-FAK (pY397) & $1: 1,000$ & $44-625 \mathrm{G}$ & $141-9$ & $\begin{array}{l}\text { Invitrogen; Thermo Fisher Scientific, Inc., } \\
\text { Waltham, MA, USA }\end{array}$ \\
\hline Goat anti-ERM & $1: 1,000$ & sc6407 & C19 & Santa Cruz Biotechnology, Inc., Dallas, TX, USA \\
\hline Mouse anti- $\gamma$-catenin & $1: 200$ & sc8415 & $\mathrm{H}-1$ & Santa Cruz Biotechnology \\
\hline Mouse anti- $\beta$-actin & $1: 2,000$ & sc47778 & $\mathrm{C} 4$ & Santa Cruz Biotechnology \\
\hline Rabbit anti-E-cadherin & $1: 300$ & sc7870 & H108 & Santa Cruz Biotechnology \\
\hline Mouse anti-kindlin-2 & $1: 200$ & MAB2617 & $3 \mathrm{~A} 3$ & EMD Millipore, Billerica, MA, USA \\
\hline $\begin{array}{l}\text { Rabbit anti-ERM[ezrin(Thr567)/ } \\
\text { radixin(Thr564)/moesin(Thr558)] }\end{array}$ & $1: 1,000$ & AB3832 & THR558 & EMD Millipore \\
\hline Mouse anti-fascin & $1: 100$ & M3567 & $55 \mathrm{~K}-2$ & Dako, Glostrup, Denmark \\
\hline Mouse anti-vimentin & $1: 500$ & M0725 & V9 & Dako \\
\hline
\end{tabular}

FAK, focal adhesion kinase; ERM, ezrin, radixin, moesin.

$(\Omega) /$ time $(\mathrm{h})$. The median \pm standard deviation rate of cell impedance for each cell line was calculated from duplicate wells. Experiments were performed in duplicate.

Cell adhesion assay. The NE2, NE3 and NEcA6 cell adhesion assays were performed as described previously (23). Briefly, the wells of 16-well E-Plates were coated with or without $10 \mu \mathrm{g} / \mathrm{ml}$ fibronectin (Sigma-Aldrich; Merck Millipore, Darmstadt, Germany) and seeded with $1 \times 10^{4}$ cells. Kindlin-2 in NE2 and NEcA6 cells were specifically knocked down by siRNA, and after $48 \mathrm{~h}$, plates were coated with $10 \mu \mathrm{g} / \mathrm{ml}$ fibronectin and seeded with $1 \times 10^{4}$ cells. Cells were monitored every 5 min using the Real Time-Cell Electronic Sensing system (Roche Applied Science) for a period of $5 \mathrm{~h}$. After plotting cell impedance against time, the rate of cell impedance (slope) was calculated using the following formula: Slope = cell impedance $(\Omega) /$ time $(\mathrm{h})$. The median \pm standard deviation rate of cell impedance for each cell line was calculated from duplicate wells and the experiment repeated twice.

Cell migration assay. The cell migration assay was performed as described previously (22). Briefly, $2 \times 10^{5}$ cells were washed once in serum-free medium, and seeded into the upper chambers of 16-well C-plates. Cell impedance was monitored as described earlier every $30 \mathrm{~min}$ for $\sim 48 \mathrm{~h}$. After plotting the cell impedance against time, the rate of cell impedance (slope) was calculated using the following formula: Slope = cell impedance $(\Omega) /$ time $(\mathrm{h})$. The median \pm standard deviation rate of cell impedance for each cell line was calculated from duplicate wells. Experiments were performed in duplicate.

Immunocytochemical analysis. Cells $\left(1 \times 10^{5}\right)$ were seeded on coverslips and incubated for $24 \mathrm{~h}$, before they were fixed in PBS containing $4 \%$ paraformaldehyde for $10 \mathrm{~min}$. After rinsing cells with PBS, they were permeabilized in $0.1 \%$ Triton $\mathrm{X}-100$ for 10 min before washing again with PBS. Non-specific binding was inhibited by incubating cells with $5 \%$ normal donkey serum (cat. no. 017-000-12; Jackson ImmunoResearch Laboratories, Inc., West Grove, PA, USA) diluted in PBS for $1 \mathrm{~h}$. The primary antibodies used in the present study are summarized in Table I. Cells were probed with an Alexa Fluor 488-conjugated Affinipure donkey anti-mouse secondary antibody (cat. no. 705-515-147; Jackson ImmunoResearch Laboratories, Inc.), and simultaneously incubated with $100 \mathrm{nM}$ Acti-stain 555 phalloidin (cat. no. PHDH1; Cytoskeleton, Inc., Denver, CO, USA), followed by counterstaining with $0.1 \mu \mathrm{g} / \mathrm{ml}$ 4',6-diamidino-2-phenylindole (cat. no. P4170; Sigma-Aldrich; Merck Millipore). Cells were analyzed using an Olympus FV1000 confocal microscope (Olympus Corporation, Tokyo, Japan).

Western blot analysis. Protein expression levels were analyzed by western blot analysis as described previously (17). Briefly, total protein cell lysates from $1 \times 10^{5}$ cells were prepared in Laemmli sample buffer (Bio-Rad Laboratories, Inc., Hercules, CA, USA). Proteins (30 $\mu \mathrm{g}$ ) were separated by $10 \%$ SDS-PAGE and transferred to $0.45 \mu \mathrm{m}$ polyvinylidene difluoride membranes (EMD Millipore, Billerica, MA, USA). The membranes were blocked in $5 \%$ non-fat milk powder in PBS containing 0.1\% Tween-20 for $1 \mathrm{~h}$ at room temperature and subsequently incubated at $4{ }^{\circ} \mathrm{C}$ overnight with primary antibodies (Table I). Membranes were then incubated with a horseradish peroxidase-conjugated goat anti-mouse secondary antibody (cat. no. sc-2005; Santa Cruz Biotechnology, Inc., Dallas, TX, USA) diluted in TBS containing $0.1 \%$ Tween-20 for $1 \mathrm{~h}$ at room temperature. Immunoreactive bands were visualized using luminol reagent (Santa Cruz Biotechnology, CA). 
Table II. Characteristics of STRs in NE2, NE3, and NEcA6 cell lines.

\begin{tabular}{lccc}
\hline & \multicolumn{3}{c}{ Cell line } \\
\cline { 2 - 4 } STR & NE2 & NE3 & NEcA6 \\
\hline D3S1358 & - & 15 & 16,18 \\
TH01 & 6,7 & 7,9 & 9 \\
D21S11 & 30,31 & $32,32.2$ & $28,32.2$ \\
D18S51 & 18 & 14,15 & 15,16 \\
Penta E & 12,20 & 12,17 & 5,11 \\
D5S818 & 12 & 12,13 & 11 \\
D13S317 & 9,12 & 8,11 & 10 \\
D7S820 & 12 & 8,12 & 11,12 \\
D16S539 & 9,11 & 10,12 & 9,12 \\
CSF1PO & 10,11 & 12 & 10,11 \\
Penta D & 9,12 & 8,12 & 10,14 \\
Amelogenin & - & - & XY \\
vWA & 14,15 & 14 & 17,19 \\
D8S1179 & - & 12,14 & 12,15 \\
TPOX & - & 11 & 7,8 \\
FGA & 21,23 & $22,25.2$ & 23,25 \\
D19S433 & - & - & $14,14.2$ \\
D2S1338 & - & - & 23,24 \\
\hline
\end{tabular}

The number of repeating units in each allele of each STR was analyzed using the PowerPlex18D System. STR, short tandem repeat.

Statistical analysis. Statistical analyses were performed in SPSS software version 13.0 (SPSS, Inc., Chicago, IL, USA). Data are expressed as the mean \pm standard deviation. Statistical significance was determined using the Student's t-test. $\mathrm{P}<0.05$ was considered to indicate a statistically significant difference.

\section{Results}

Characteristics of STRs in NE2, NE3, and NEcA6 cells. To the best of our knowledge, the present study is the first to characterize STRs in the NE2, NE3, and NEcA6 esophageal epithelial cell lines (Table II), which were immortalized by transfection with hTERT and/or human HPV 16 E6/E7. The detailed differences in genetic characterization are presented in Table II.

$N E c A 6$ cells were transformed and exhibited a more aggressive tumorigenic phenotype in vitro and in vivo when compared with NE2 and NE3 cells. Previous studies have reported that NE2, NE3 and NEcA6 immortalized cell lines were highly proliferative, but failed to induce colony formation in soft agar $(19,20,24)$. These observations were verified using the xCELLigence RTCA system and soft agar colony formation assays in the present study. As shown in Fig. 1A, the proliferation rate of NE3 cells and NEcA6 cells was significantly higher when compared with NE2 cells during the 40 -h period after cell seeding $(\mathrm{P}=0.043)$. NE2 cells and NE3 cells were unable to grow in soft agar, whereas NEcA6 cells formed small colonies (Fig. 1B), indicating that the NEcA6 cells may have acquired the capacity for
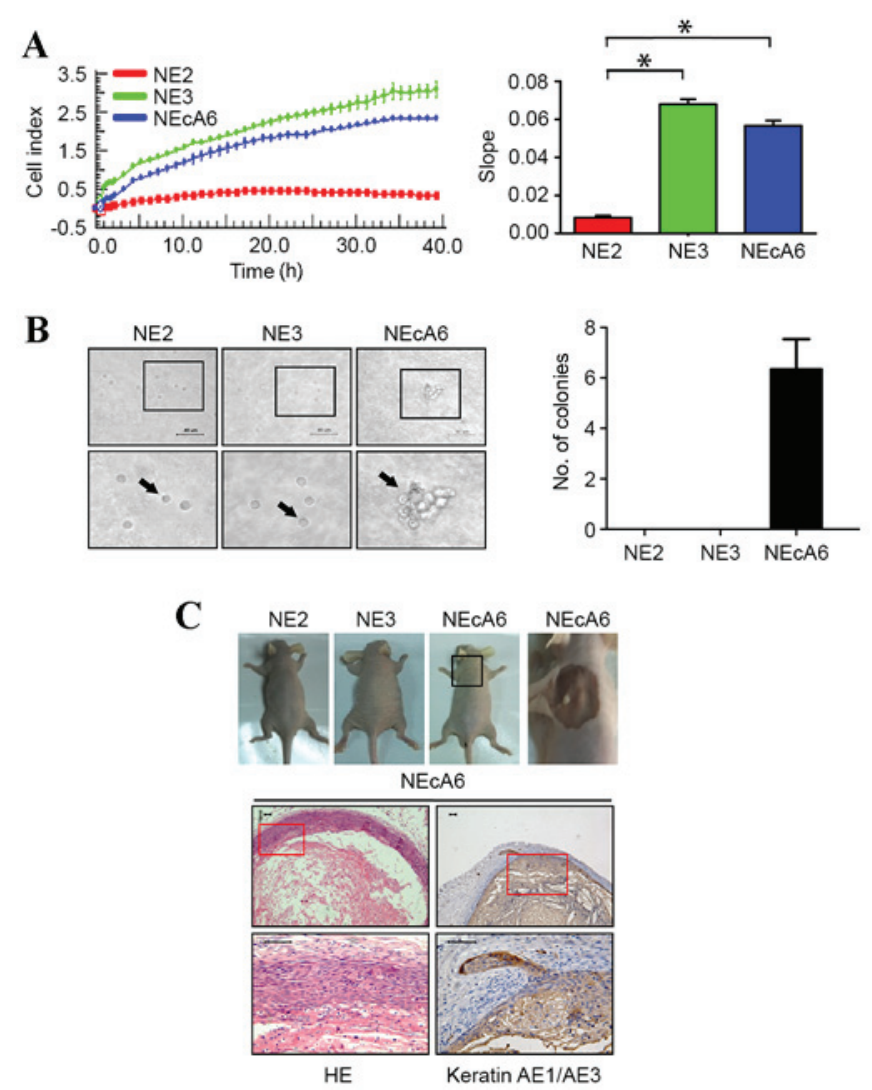

Figure 1. Proliferation and tumorigenic phenotype of immortalized esophageal epithelial cell lines NE2, NE3 and NEcA6 in vitro and in vivo. (A) The proliferation profile of NE2, NE3 and NEcA6 cells. Real-time detection of impedance response/cell index curves determined over $48 \mathrm{~h}$. The bar chart shows the slope [cell impedance $(\Omega) /$ time $(\mathrm{h})$ ] of the cell proliferation profiles. ${ }^{*} \mathrm{P}<0.05$ vs. NE2 cells. (B) Soft-agar colony formation assay of NE2, NE3 and NEcA6 cells over the course of 3 weeks (magnification, $x 40$; scale bars, $40 \mu \mathrm{m}$ ). Black arrows indicate the cells in agar. The bar chart shows the number of colonies formed for each cell line. Data are presented as the mean \pm standard deviation $(n=3)$. (C) Tumorigenicity assays in nude mice. NE2, NE3, and NEcA6 cells were injected into $\mathrm{Nu} / \mathrm{Nu}$ mice ( $1 \times 10^{6}$ cells/flank). At 35 days following injection, tumors were removed (upper panel). HE staining and immunohistochemical staining of cytokeratin in the NEcA6 xenografted tumors (lower panel; scale bars, $50 \mu \mathrm{m}$ ). HE, hematoxylin-eosin.

anchorage-independent growth during the passaging process. To further test the tumorigenicity of these cell lines in vivo, they were injected subcutaneously into nude mice. As shown in Fig. 1C, NE2 cells and NE3 cells failed to form tumors, whereas two out of six sites injected with NEcA6 cells formed tumors $\left(\sim 5 \mathrm{~mm}^{3}\right.$ in volume) 35 days after xenografting. Immunohistochemical staining demonstrated that cytokeratins, which are markers for epithelial tissues, were expressed in NEcA6-generated xenografts (Fig. 1C). These results were confirmed using an additional five $\mathrm{Nu} / \mathrm{Nu}$ mice, of which 10 out of 20 NEcA6 implanted sites formed tumors $\left(\sim 5 \mathrm{~mm}^{3}\right.$ in volume; data not shown). These data suggest that NEcA6 were transformed and exhibit a more aggressive tumorigenic phenotype in vitro and in vivo compared with NE2 and NE3 cells.

NEcA6 cells migrated further than NE2 and NE3 cells. Cancer cell migration is known to serve a major role in the progression of malignant tumors. Therefore, the migration capabilities of NE2, NE3 and NEcA6cell lines were examined using the xCELLigence 

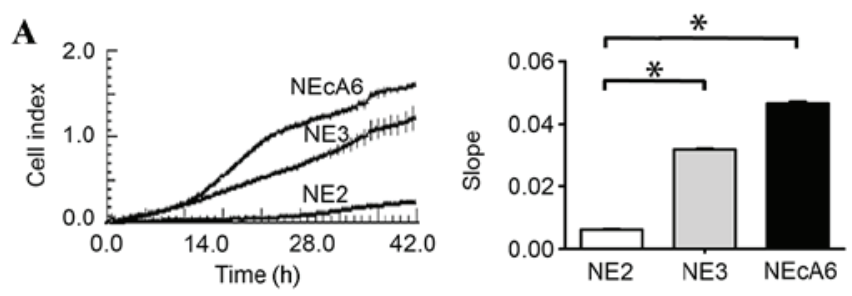

B
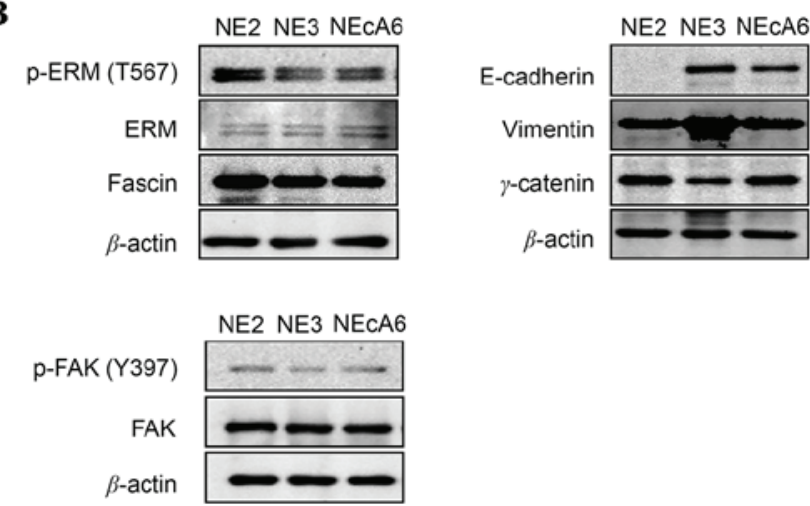

Figure 2. Migration capabilities of the NE2, NE3 and NEcA6 immortalized esophageal epithelial cell lines. (A) Migration profiles of NE2, NE3, and NEcA6 cells. Real-time detection of impedance response/cell index curves determined over $48 \mathrm{~h}$. The bar chart shows the slope [cell impedance $(\Omega) /$ time $(\mathrm{h})]$ of the cell migration profiles. Data are presented as the mean \pm standard deviation $(n=2)$. ${ }^{*} \mathrm{P}<0.05$ vs. NE2 cells. (B) Western blot analysis of migration-related proteins and biomarkers of the epithelial-mesenchymal transition in the three cell lines. $\beta$-actin was used as a loading control. pERM, phosphorylated ezrin, radixin, moesin; FAK, focal adhesion kinase.

RTCA system. As shown in Fig. 2A, the migration capabilities of NEcA6 cells were significantly higher when compared to that of the NE2 and NE3 cells, which was consistent with the results of the tumorigenicity assay. In order to investigate the mechanisms underlying the prominent migration ability of NEcA6 cells, the expression levels of fascin and ezrin-radixin-moesin (ERM), as well as their activated forms, which have been demonstrated to serve important roles in the migration of esophageal carcinoma cells $(25,26)$, were examined. As shown in Fig. 2B, no significant differences in the protein expression levels of fascin and ERM proteins were observed among the three cell lines. In addition, no significant differences in the protein expression levels of FAK and phosphorylated (p)-FAK (Tyr397) were observed among the three cell lines (Fig. 2B). FAK and p-FAK are known to regulate several signaling pathways that lead to cell proliferation, migration, and adhesion (27). The protein expression levels of epithelial and mesenchymal biomarkers vimentin, $\gamma$-catenin and E-cadherin in these cells were then examined. Notably, high expression levels of vimentin and $\gamma$-catenin were detected in all cell lines, however, NE2 cells lacked detectable levels of E-cadherin (Fig. 2B). This indicates that NE2 cells may have undergone an epithelial-mesenchymal transition (EMT)-like process, which is consistent with results of a previous study (24).

Immunocytochemical staining of kindlin, talin and paxillin reveals typical dot-like focal adhesions located at the distal ends of stress fibers in NEcA6 cells. Cell proliferation, migration and adhesion depend on cell-to-cell and cell-to-ECM

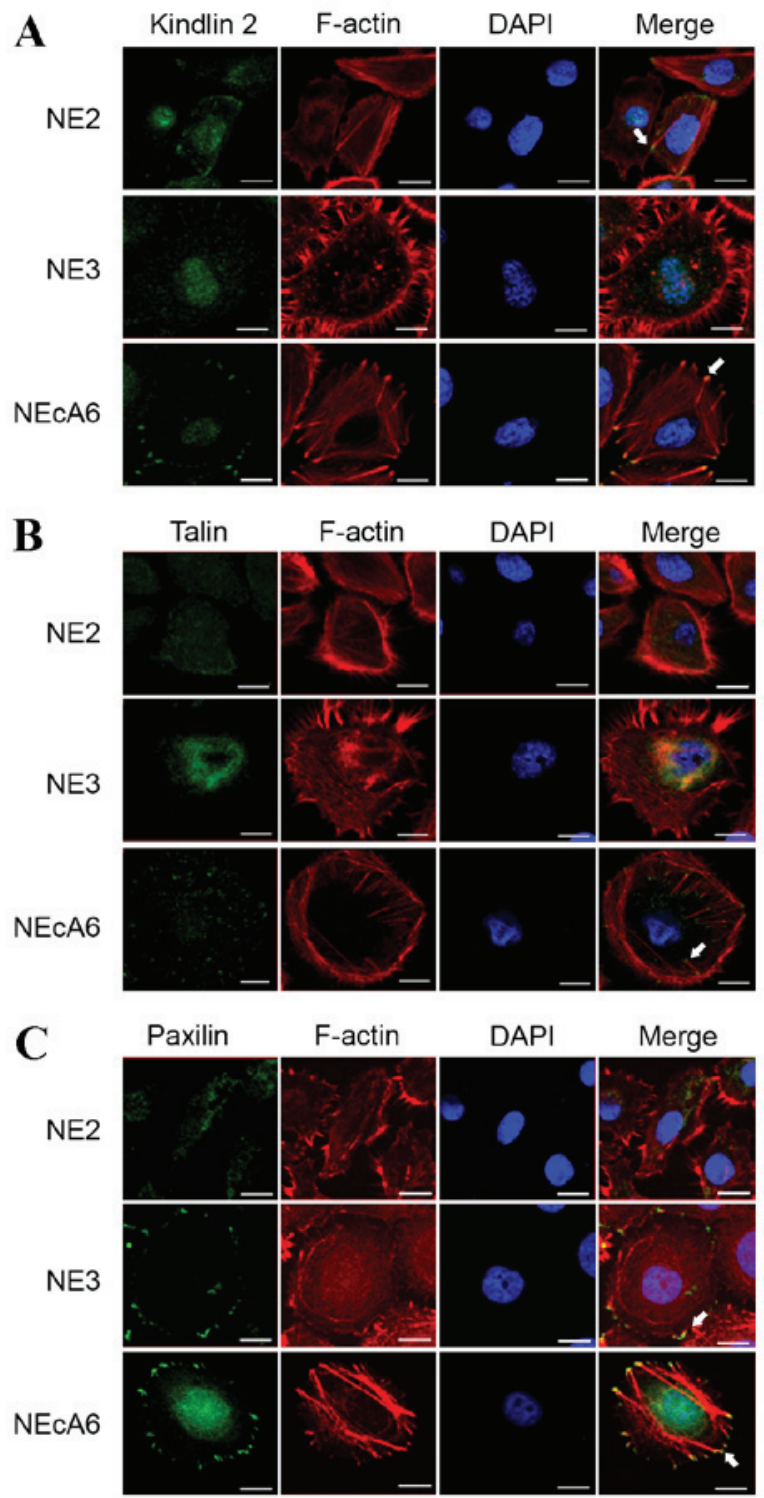

Figure 3. Localization of kindlin, talin, and paxillin in NE2, NE3 and NEcA6 immortalized esophageal epithelial cell lines. NE2, NE3, and NEcA6 cells were cultured on slides for $24 \mathrm{~h}$, fixed and stained for the predominant components of focal adhesions (A) kindlin, (B) talin, and (C) paxillin (green fluorescent signals), as well as for actin microfilaments (red fluorescent signals). Merged images show the co-localization of focal adhesions and actin microfilaments (yellow fluorescent signals), as indicated by white arrows. Images obtains by confocal microscopy (FV1000; Olympus) equipped with a 60x, 1.42NA, Plan oil objective (Olympus, Japan) Scale bar, $20 \mu \mathrm{m}$. DAPI, 4',6-diamidino-2-phenylindole.

interactions, and the focal adhesion receptors kindlin, talin and paxillin serve essential roles in these processes $(9,14)$. Therefore, the expression and subcellular localization of these focal adhesion markers, as well as their co-localization with F-actin were examined in NE2, NE3 and NEcA6 cells using immunofluorescence assays (Fig. 3). Kindlin-2 was localized to the nucleus in all cell lines (Fig. 3A). Kindlin-2 was also present in focal adhesions in NEcA6 cells, where it was co-localized with the termini of actin stress fibers (Fig. 3A). By contrast, NE2 and NE3 cells exhibited reduced kindlin-2 expression at the distal ends of stress fibers (Fig. 3A). Talin was present in the typical punctate structures of focal adhesions and distributed in the protrusions of NEcA6 cells, whereas 
A

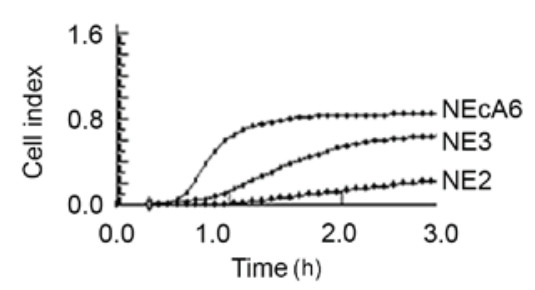

B

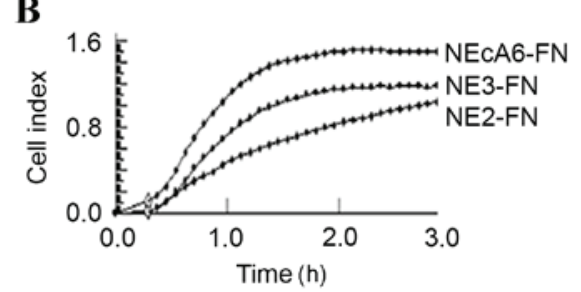

C

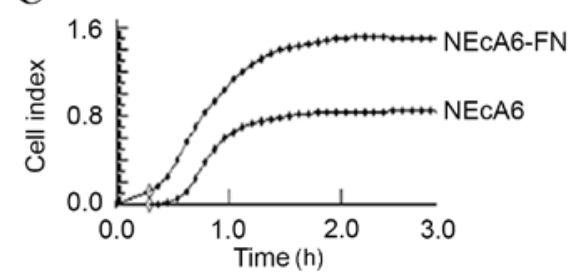

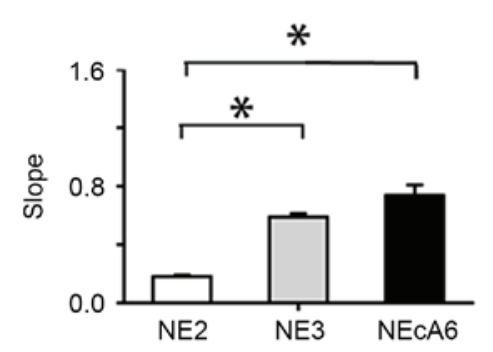
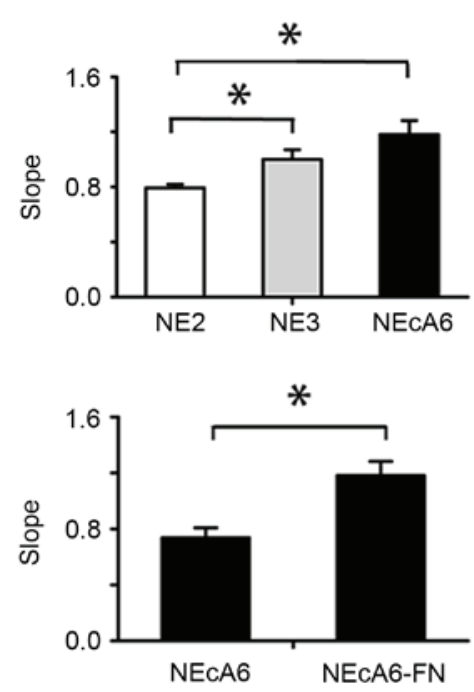

Figure 4. Quantitative and dynamic monitoring of NE2, NE3 and NEcA6 cell adhesion and migration in response to FN-coated surfaces. Cells were plated on E-plates in the (A) absence or (B) presence of $10 \mu \mathrm{g} / \mathrm{ml} \mathrm{FN}$. Cell adherence and migration was measured over the course of $3 \mathrm{~h}$. The cell index indicates the degree of cell adhesion. (C) A summary of the adhesion and migration capabilities of NEcA6 cells. Bar charts show the slope [cell impedance ( $\Omega$ )/time (h)] of the cell migration profiles. Data are expressed as the mean \pm standard deviation $(n=2)$. ${ }^{*} \mathrm{P}<0.05$ vs. NE2 or NEcA6 cells. FN, fibronectin.

talin staining in NE2 cells and NE3 cells was diffuse (Fig. 3B). Low talin expression was observed in the perinuclear region of NE3 cells (Fig. 3B). As presented in Fig. 3C, paxillin was dispersed in the cytoplasm of NE2 cells, and was present at the edge of NE3 cells. By contrast no detectable co-localization of paxilin and F-actin was observed in all cell lines examined (Fig. 3C). Paxillin was observed in the typical focal adhesions of NEcA6 cells, as indicated by strong staining at the termini of thick stress fibers. In addition, paxillin was localized at the nucleus and perinuclear regions in NEcA6 cells (Fig. 3C). Notably, kindlin-2, talin and paxillin were present at sites of focal adhesion and localized at the two distal terminals of stress fibers in NEcA6 cells, suggesting that NEcA6 cells form a greater number of mature focal adhesions compared with the other two cell lines (Fig. 3). These features may be associated with increased cell proliferation and migration, which may lead to a more aggressive tumorigenic phenotype in NEcA6 cells, compared with NE2 and NE3 cells.

NEcA6 cells exhibit increased adhesion capabilities when compared with NE2 and NE3 cells. Cells adhere to the ECM via focal contacts, which mature into focal adhesions containing kindlin, talin, and paxillin $(13,14,28)$. Cell adhesion depends on ECM interactions (29-31). The integrin protein family and focal adhesion proteins serve essential roles during this process by interacting with ECM proteins, such as fibronectin and the actin-based cytoskeleton $(29,30,32)$. It was therefore hypothesized that the larger number of mature focal contacts in NEcA6 cells may lead to abnormal cell adhesion, proliferation and migration. In order to test this hypothesis, the degree of cell adhesion and migration of all cell lines with and without fibronectin was examined using the xCELLigence RTCA system. As shown in Fig. 4, NEcA6 cells exhibited significantly higher $(\mathrm{P}=0.002)$ adhesion capabilities when compared with NE2 and NE3 cell lines in the presence and absence of fibronectin. Cell adhesion was maximal at $2 \mathrm{~h}$ after the cells were seeded (Fig. 4). The seeding of all cell lines onto fibronectin-coated wells led to a marked increase in the cell index (indicative of increased cell adhesion) when compared with cells seeded on plates without fibronectin (Fig. 4).

siRNA-mediated depletion of kindlin-2 leads to a greater decrease in cell adhesion capabilities in NEcA6 cells than NE3 cells when compared with non-targeting controls. The above results demonstrate that the focal adhesion receptors kindlin, talin, and paxillin exhibited typical focal adhesion morphology in NEcA6 cells, which was different from the other two cell lines. These alterations may be responsible for the observed alterations in cell migration, adhesion and proliferation among the cell lines. In order to investigate this further, the effect of siRNA-mediated depletion of kindlin-2, which has been demonstrated to regulate cell-to-ECM interactions, induce the formation of focal adhesions and promote cancer cell adhesion $(23,25)$, was examined in NE3 and NEcA6 cells. Western blotting results revealed that kindlin-2 siRNA-treated cells demonstrated a marked reduction in kindlin-2 protein 
expression levels when compared with the negative control cells (Fig. 5A). The effect of kindlin-2 depletion on cell adhesion and migration was then evaluated using the xCELLigence RTCA system. siRNA-treated NE3 and NEcA6 cells exhibited a significant reduction $(\mathrm{P}=0.003)$ in cell adhesion when compared with negative control cells (Fig. 5B). Notably, the adhesion rates of siRNA-treated NE3 and NEcA6 cells were reduced by 42 and $66 \%$, respectively, when compared with the negative controls. In addition, the adhesion rate of NEcA6 cells was decreased by $24 \%$ compared with that of NE3 cells (Fig. 5B and C). These results indicate that the effect of kindlin-2 knockdown on cell adhesion capabilities was greater in NEcA6 cells when compared with that of NE3 cells. Therefore, the authors hypothesize that kindlin-2 may contribute to the carcinogenesis of NEcA6 cells.

\section{Discussion}

Immortalized esophageal epithelial cell lines derived from Chinese patients are currently scarce $(18,24,33)$. To the best of our knowledge, the present study is the first to characterize the STRs of NE2, NE3 and NEcA6 immortalized esophageal epithelial cell lines. This characterization may serve as the foundation for the genetic identification of these cell lines in future studies. Unexpectedly, our in vitro and in vivo investigations revealed that NEcA6 cells formed small dense colonies in soft agar and small tumors in nude mice, indicating that these cells may have undergone transformation. Compared with NE2 and NE3 cells, NEcA6 cells exhibited a more aggressive tumorigenic phenotype in vitro and in vivo. Based on these results, it is formally possible that cytogenetic aberrations occurred during the progressive passaging of these cells (18). However, the molecular/epigenetic events underlying the more aggressive tumorigenic phenotype in NEcA6 cells remain unknown. In addition, NEcA6 cells demonstrated increased migration capabilities when compared with NE2 and NE3 cells. However, no significant alterations in the expression levels of migration-related proteins fascin, ERM, and p-FAK (Tyr397) were observed among the cell lines. Furthermore, NE2 cells appeared to have undergone an EMT-like process, whereas NE3 cells and NEcA6 cells expressed both E-cadherin and vimentin. These findings suggest that additional mechanisms may have been responsible for the high migration ability of NEcA6 cells.

The expression and subcellular localization of kindlin-2, talin, and paxillin was investigated further using immunofluorescence techniques. Notably, these focal adhesion components exhibited a typical morphology and were located at the two distal terminals of stress fibers in NEcA6 cells, but not in NE2 or NE3 cells. The focal adhesion protein paxillin functions as a multi-domain adapter molecule, and is a target of many oncogenes such as $\mathrm{v}$-Src and the human papillomavirus E6 protein $(19,34)$. In addition, paxillin recruits actin to its C-terminus at sites of cell adhesion to the ECM, and undergoes extensive phosphorylation during integrin-mediated cell adhesion $(35,36)$. Therefore, paxillin is considered to function at the crossroads of cell adhesion and cell migration $(13,37)$. Similarly, talin and kindlin, which are two protein families consisting of four point one, ezrin, radixin, moesin-domain proteins, are components of focal adhesions that link the
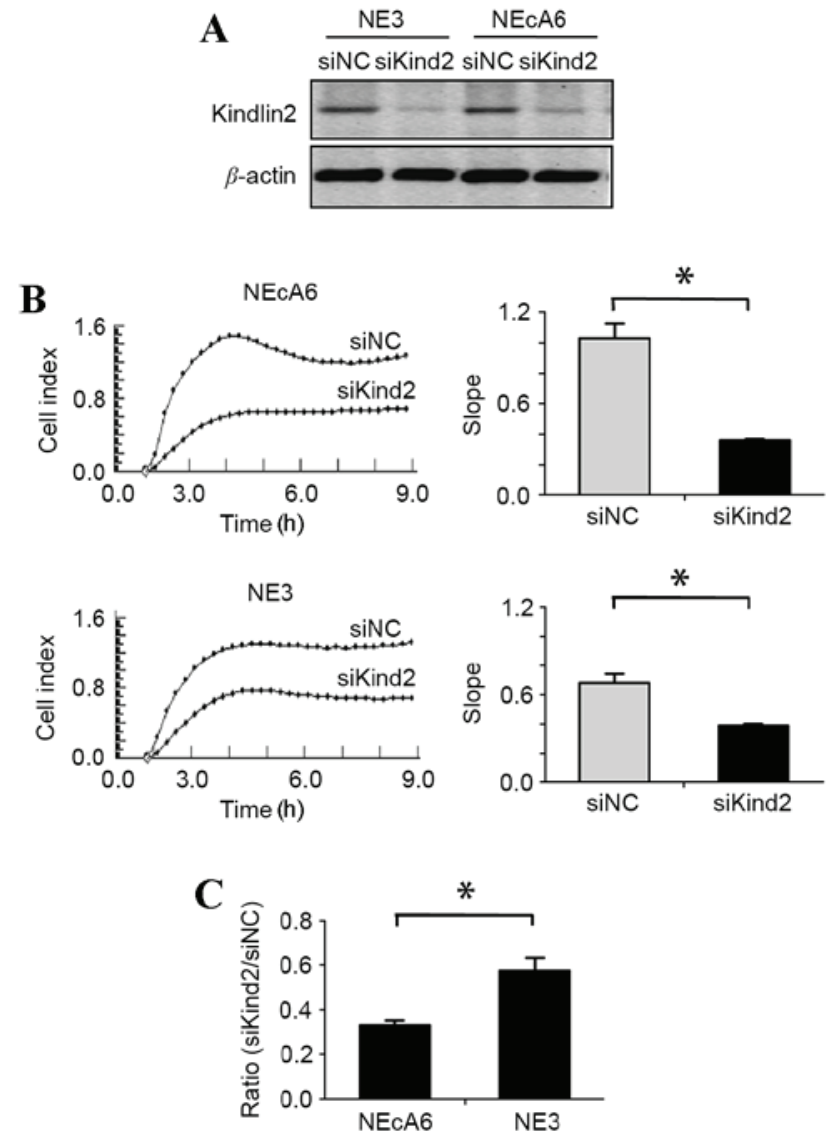

Figure 5. Inhibition of cell adhesion by siRNA-mediated knockdown of kindlin-2 in NE3 and NEcA6 cells. (A) Western blot analysis of kindlin-2 expression in NE2 and NEcA6 cells following transfection with kindlin-2 siRNA or non-targeting controls. $\beta$-actin was used as a loading control. (B) Adhesion profiles of NE3 and NEcA6 cells following siRNA-mediated knockdown of kindlin-2. The cells were plated on E-plates with $5 \mu \mathrm{g} / \mathrm{ml}$ fibronectin, and cell adhesion and migration was measured over the course of $9 \mathrm{~h}$. (C) The arithmetic mean of the sikind2/siNC cell index ratio for NE3 and NEcA6 cell lines. The ratio (sikind2/siNC) represents the decreased ability of adhesion by kindling- 2 knockdown in NE3 and NEcA6 cells. Bar charts show the slope [cell impedance $(\Omega) /$ time $(\mathrm{h})$ ] of the cell migration profiles. Data are presented as the mean \pm standard deviation $(n=2)$. ${ }^{*} \mathrm{P}<0.05$ vs. siNC or NEcA6 cells. siRNA, small interfering RNA; NC, negative control.

ECM to the actin cytoskeleton by binding directly to the cytoplasmic tails of $\beta$-integrin subunits (9). These proteins also serve a key role in modulating integrin activity, and transduce signals primarily through the inside-out activation of integrin signaling pathways $(14,38)$. The detailed mechanisms underlying the effects of kindlin proteins on integrin activation remain unclear, however, it is clear that these proteins cooperate with talin to activate integrin by binding to the cytoplasmic tails of $\beta 1$ and $\beta 3$ integrins $(34,39)$. In the present study, these three crucial components of focal adhesions exhibited a typical dot-like staining in NEcA6 cells, indicative of the formation of a large number of focal contacts. Formation of these adhesion contacts depends on FAK and integrin, and these adhesions stabilize the lamellipodia by mediating attachment to the ECM and the rearrangement of actin, thereby contributing to efficient migration (32). In addition to the modulation of integrin-mediated cell migration, these three focal adhesion markers have been demonstrated to regulate cell adhesion by affecting the recruitment of focal 
adhesion complex components (14,37). Adhesion of fibroblasts to fibronectin stimulates the phosphorylation of tyrosine in paxillin by FAK, which is a signal transduction mechanism associated with cell adhesion and cytoskeletal reorganization (37). Therefore, the authors hypothesize that NEcA6 cells not only possess prominent migration ability, but also display high adhesion ability. Indeed NEcA6 cells demonstrated the highest adhesion capabilities in the absence of ECM and in fibronectin-coated wells among all cell lines examined.

An increasing number of studies have demonstrated the importance of kindlin-2 in the malignant progression of tumors (40-43). A recent study reported that kindlin-2 is highly expressed in the invasive edge of tumors, and its overexpression promotes cell migration/invasion $(34,44)$. However, the expression and biological significance of kindlin-2 in immortalized esophageal epithelial cells is unclear. The results of the present study revealed that kindlin- 2 was expressed in NE3 and NEcA6 cells, and siRNA-mediated knockdown of kindlin-2 reduced the adhesion capabilities of these cell lines. Compared with NE3 cells, knockdown of kindlin-2 in NEcA6 cells was associated with a greater reduction in cell adhesion, which suggests that kindlin-2 may serve a role in the tumorigenic phenotype displayed by these cells. However, kindlin-2 knockdown did not completely inhibit cell adhesion in these cell lines, which suggests that additional regulatory mechanisms may be involved in mediating carcinogenesis. Further studies are required to validate this mechanism in the cell lines.

In conclusion, the findings of the present study reveal the migration and adhesion characteristics of NE2, NE3 and NEcA6 immortalized esophageal epithelial cell lines, and demonstrate the distribution of kindlin-2, talin and paxillin. The results suggest that these cell lines may be valuable models for future studies investigating the processes of cell migration and adhesion, as well as the molecular mechanisms that contribute to esophageal carcinogenesis. Of particular note, the transformed NEcA6 cells may be useful in future studies involving the investigation of cytogenetic alterations that occur in the initial stages of carcinogenesis in vitro.

\section{Acknowledgments}

The authors would like to thank Professor Sai-Wah Tsao (Department of Anatomy, University of Hong Kong, Hong, Kong, China) for supplying the NE2, NE3, and NEcA6 cells used for the purposes of this study. In addition, the authors would like to thank Dr Wei-Jiang Zhao, (Neural Science Center, Shantou University Medical College, Shantou, China) for assisting with the completion of the confocal microscopy analysis. The present study was supported by grants from the Natural Science Foundation of China-Guangdong Joint Fund (grant nos. U1301227 and U0932001) and the National Natural Science Foundation of China (grant nos. 81172264 and 81472613).

\section{References}

1. Vicente-Manzanares M, Webb DJ and Horwitz AR: Cell migration at a glance. J Cell Sci 118: 4917-4919, 2005.
2. Axelrad JE, Lichtiger S and Yajnik V: Inflammatory bowel disease and cancer: The role of inflammation, immunosuppression, and cancer treatment. World J Gastroenterol 22: 4794-4801, 2016.

3. Mayor R and Etienne-Manneville S: The front and rear of collective cell migration. Nat Rev Mol Cell Biol 17: 97-109, 2016.

4. Ridley AJ, Schwartz MA, Burridge K, Firtel RA, Ginsberg MH, Borisy G, Parsons JT and Horwitz AR: Cell migration: Integrating signals from front to back. Science 302: 1704-1709, 2003.

5. Etienne-Manneville S: Adherens junctions during cell migration. Subcell Biochem 60: 225-249, 2012.

6. Turner CE: Paxillin. Int J Biochem Cell Biol 30: 955-959, 1998.

7. Friedl $\mathrm{P}$ and Wolf $\mathrm{K}$ : Tumour-cell invasion and migration: Diversity and escape mechanisms. Nat Rev Cancer 3: 362-374, 2003.

8. Kim DH and Wirtz D: Focal adhesion size uniquely predicts cell migration. FASEB J 27: 1351-1361, 2013.

9. Clark EA and Brugge JS: Integrins and signal transduction pathways: The road taken. Science 268: 233-239, 1995.

10. Turner CE, Glenney JR Jr and Burridge K: Paxillin: A new vinculin-binding protein present in focal adhesions. J Cell Biol 111: 1059-1068, 1990.

11. Brown MC and Turner CE: Paxillin: Adapting to change. Physiol Rev 84: 1315-1339, 2004.

12. Sheppard D: In vivo functions of integrins: Lessons from null mutations in mice. Matrix Biol 19: 203-209, 2000

13. Turner CE: Paxillin and focal adhesion signalling. Nat Cell Biol 2: E231-E236, 2000.

14. Calderwood DA, Campbell ID and Critchley DR: Talins and kindlins: Partners in integrin-mediated adhesion. Nat Rev Mol Cell Biol 14: 503-517, 2013.

15. Wang H, Spillare EA, Wang QS, Sabourin CLK and Stoner GD: p53-independent down-regulation of cyclin D1 and p21Waf1 in the process of immortalization of human esophageal epithelial cells. Int J Oncol 12: 325-328, 1998.

16. Shen Z, Cen S, Shen J, Cai W, Xu J, Teng Z, Hu Z and Zeng Y: Study of immortalization and malignant transformation of human embryonic esophageal epithelial cells induced by HPV18 E6E7. J Cancer Res Clin Oncol 126: 589-594, 2000.

17. Sashiyama H, Shino Y, Kawamata Y, Tomita Y, Ogawa N, Shimada H, Kobayashi S, Asano T, Ochiai T and Shirasawa H: Immortalization of human esophageal keratinocytes by E6 and E7 of human papillomavirus type 16. Int J Oncol 19: 97-103, 2001.

18. Zhang H, Jin Y, Chen X, Jin C, Law S, Tsao SW and Kwong YL: Cytogenetic aberrations in immortalization of esophageal epithelial cells. Cancer Genet Cytogenet 165: 25-35, 2006.

19. Fu L, Qin YR, Xie D, Hu L, Kwong DL, Srivastava G, Tsao SW and Guan XY: Characterization of a novel tumor-suppressor gene PLC delta 1 at 3p22 in esophageal squamous cell carcinoma. Cancer Res 67: 10720-10726, 2007.

20. Zhuang ZH, Tsao SW, Deng W, Wang JD, Xia HH, He H, Feng HC, Wang LD, Gu Q, Lam SK, et al: Early upregulation of cyclooxygenase-2 in human papillomavirus type 16 and telomerase-induced immortalization of human esophageal epithelial cells. J Gastroenterol Hepatol 23: 1613-1620, 2008.

21. Zhao Q, Shen JH, Shen ZY, Wu ZY, Xu XE, Xie JJ, Wu JY, Huang Q, Lu XF, Li EM and Xu LY: Phosphorylation of fascin decreases the risk of poor survival in patients with esophageal squamous cell carcinoma. J Histochem Cytochem 58: 979-988, 2010.

22. Limame R, Wouters A, Pauwels B, Fransen E, Peeters M, Lardon F, De Wever O and Pauwels P: Comparative analysis of dynamic cell viability, migration and invasion assessments by novel real-time technology and classic endpoint assays. PLoS One 7: e46536, 2012.

23. Atienza JM, Zhu J, Wang X, Xu X and Abassi Y: Dynamic monitoring of cell adhesion and spreading on microelectronic sensor arrays. J Biomol Screen 10: 795-805, 2005.

24. Cheung PY, Deng W, Man C, Tse WW, Srivastava G, Law S, Tsao SW and Cheung AL: Genetic alterations in a telomerase-immortalized human esophageal epithelial cell line: Implications for carcinogenesis. Cancer Lett 293: 41-51, 2010.

25. Xie JJ, Xu LY, Zhang HH, Cai WJ, Mai RQ, Xie YM, Yang ZM, Niu YD, Shen ZY and Li EM: Role of fascin in the proliferation and invasiveness of esophageal carcinoma cells. Biochem Biophys Res Commun 337: 355-362, 2005.

26. Xie JJ, Xu LY, Xie YM, Zhang HH, Cai WJ, Zhou F, Shen ZY and $\mathrm{Li}$ EM: Roles of ezrin in the growth and invasiveness of esophageal squamous carcinoma cells. Int J Cancer 124: 2549-2558, 2009. 
27. Mitra SK, Hanson DA and Schlaepfer DD: Focal adhesion kinase: In command and control of cell motility. Nat Rev Mol Cell Biol 6: 56-68, 2005

28. Rondas D, Tomas A and Halban PA: Focal adhesion remodeling is crucial for glucose-stimulated insulin secretion and involves activation of focal adhesion kinase and paxillin. Diabetes 60: 1146-1157, 2011.

29. Baumann K: Cell adhesion: Extracellular bonds. Nat Rev Mol Cell Biol 14: 404, 2013.

30. Wrighton KH: Cell adhesion: The 'ins' and 'outs' of integrin signalling. Nat Rev Mol Cell Biol 14: 752, 2013.

31. Multhaupt HA, Leitinger B, Gullberg D and Couchman JR: Extracellular matrix component signaling in cancer. Adv Drug Deliv Rev 97: 28-40, 2016.

32. Carragher NO and Frame MC: Focal adhesion and actin dynamics: A place where kinases and proteases meet to promote invasion. Trends Cell Biol 14: 241-249, 2004.

33. Yu HP, Xu SQ, Liu L, Shi LY, Cai XK, Lu WH, Lu B, Su YH and Li YY: Cyclooxygenase-2 expression in squamous dysplasia and squamous cell carcinoma of the esophagus. Cancer Lett 198: 193-201, 2003

34. An Z, Dobra K, Lock JG, Strömblad S, Hjerpe A and Zhang H: Kindlin-2 is expressed in malignant mesothelioma and is required for tumor cell adhesion and migration. Int J Cancer 127: 1999-2008, 2010.

35. Deakin NO and Turner CE: Paxillin comes of age. J Cell Sci 121: 2435-2444, 2008.

36. Lawson C and Schlaepfer DD: Integrin adhesions: Who's on first? What's on second? Connections between FAK and talin. Cell Adh Migr 6: 302-306, 2012.
37. Schaller MD: Paxillin: A focal adhesion-associated adaptor protein. Oncogene 20: 6459-6472, 2001.

38. Karaköse E, Schiller HB and Fässler R: The kindlins at a glance. J Cell Sci 123: 2353-2356, 2010.

39. Liao Z, Kato H, Pandey M, Cantor JM, Ablooglu AJ, Ginsberg MH and Shattil SJ: Interaction of kindlin- 2 with integrin $\beta 3$ promotes outside-in signaling responses by the $\alpha \mathrm{V} \beta 3$ vitronectin receptor. Blood 125: 1995-2004, 2015.

40. Shen Z, Ye Y, Kauttu T, Seppänen H, Vainionpää S, Wang S, Mustonen $\mathrm{H}$ and Puolakkainen P: Novel focal adhesion protein kindlin-2 promotes the invasion of gastric cancer cells through phosphorylation of integrin $\beta 1$ and $\beta 3$. J Surg Oncol 108: 106-112, 2013.

41. Zhang HF, Zhang K, Liao LD, Li LY, Du ZP, Wu BL, Wu JY, Xu XE, Zeng FM, Chen B, et al: miR-200b suppresses invasiveness and modulates the cytoskeletal and adhesive machinery in esophageal squamous cell carcinoma cells via targeting Kindlin-2. Carcinogenesis 35: 292-301, 2014

42. Ge YS, Liu D, Jia WD, Li JS, Ma JL, Yu JH and Xu GL: Kindlin-2: A novel prognostic biomarker for patients with hepatocellular carcinoma. Pathol Res Pract 211: 198-202, 2015.

43. Ren Y, Jin H, Xue Z, Xu Q, Wang S, Zhao G, Huang J and Huang H: Kindlin-2 inhibited the growth and migration of colorectal cancer cells. Tumour Biol 36: 4107-4114, 2015.

44. Yu Y, Wu J, Wang Y, Zhao T, Ma B, Liu Y, Fang W, Zhu WG and Zhang $\mathrm{H}$ : Kindlin 2 forms a transcriptional complex with $\beta$-catenin and TCF4 to enhance Wnt signalling. EMBO Rep 13: 750-758, 2012. 